\title{
Energy saving in P2P oriented Wireless Sensor Network (WSN) using the approach of various compression techniques
}

\author{
${ }^{1}$ Archita Dad, ${ }^{2}$ Charika Jain, ${ }^{3}$ Anil Saroliya \\ 1,2,3 Amity School of Engg \& Tech., Amity University Rajasthan
}

\begin{abstract}
Recent advancements \& popularity of peer-to- peer networks draws a lot of attention of networking researchers. Wireless sensor network (WSN) consists of various scattered autonomous sensors to maintain regular supervision on physical or environmental conditions, such as temperature, vibration, audible signals, pressure, movements and to collaboratively pass their data through the network to a main location. So in this paper we have considered energy saving concept of wireless sensor network with respect to P2P network technology. For reducing energy consumption by sensors the use of various compression techniques on data before transmission have discussed in this paper.

Different types of compression techniques are there and the best one is dependent on the network technology and data characteristics. For choosing the best one of them we have discussed Adaptive Compression Environment technology (ACE) in this paper.

Keywords: Peer-To-Peer (P2P) Networks, Wireless Sensor Networks (WSN), Data Compression Techniques, Distributed Networks, Adaptive Compression Environment (ACE)
\end{abstract}

\section{Introduction}

A large no of sensing devices which have limited computing power and radio communication, capabilities build up a sensor network. Sensor senses any changes in environment and as it occurs it does some operation. Although sensor may be mobile they can be recognized stationary after deployment.

A network configuration comprises of sensor working unattended and transmitting their observation values to some processing. Due to limited transmission range, sensors convey their data through multi-hope communication i.e. using intermediate nodes as relays. In this case the sensor may be both a data source and a data router.

In many of the applications sensor networks have battery-power nodes with inadequate energy recourses. It is inconvenient to replace or recharge sensors battery in its working environment. Thus when the energy of node exhausts, it stops sensing or routing data so the network performance degrades. This implies that making good uses of energy resources is a necessity in sensor networks.

\section{Energy Saving in WSN}

Resent advance in internet technology volume of data transmitted by peer to peer system increases, due to this the energy consumption of sensors increases so to reduce the energy consumption various techniques are used such as energy-efficient MAC layer schemes, Traffic routing and connectivity, energy aware strategies for data dissemination and data collection [1].

From the energy saving view point, a low power operational mode is introduced, the so called sleep mode, during idle periods. Sensor works in two major operational states: active and sleep. The active state includes three operational modes: transmit, receive, and idle. In the transmitting mode, energy is spent in the front-end amplifier that supplies the power for the actual RF transmission. In the receiving mode, energy is consumed entirely by the transceiver electronics and by processing functions, such as demodulation and decoding. In the idle state, a node typically listens to the wireless channel without actively receiving. In idle mode, energy expenditure is mainly due to processing activity, since the voltage controlled oscillator is functioning and all circuits are maintained ready to operate.

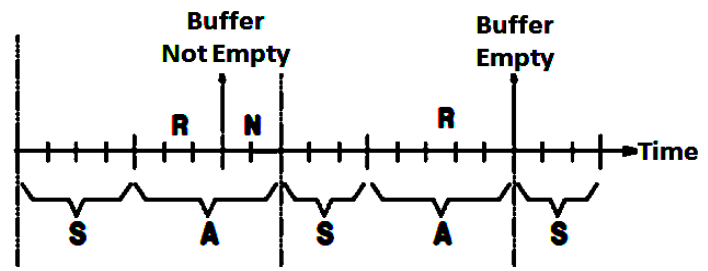

Figure 1: States of Sensor [2] 
Based on the above observations, we describe the temporal evolution Et of the sensor state in terms of cycles, as depicted in Figure 1. Each cycle comprises a sleep phase (S) and an active phase (A). During phase $\mathrm{S}$, the sensor is in sleep mode. When the sensor switches to the active mode, phase A begins and the sensor schedules a time instant in the future at which it will go back to sleep. However, at the time slot at which the sensor should go to sleep, its data buffer may not be empty. So the active phase can thus be divided into an initial phase $(\mathrm{R})$ and (possibly) a phase $(\mathrm{N})$. In phase $\mathrm{R}$ the sensor can receive and transmit; also it generates data. In phase $\mathrm{N}$ the sensor does not receive nor generate data; it can only transmit the data units that are still in its buffer or be idle waiting for a transmission opportunity [2].

\section{Transmission in Various Models}

The main motive of using this sensor technology is for receiving / transmitting of packets. For transmitting and receiving of data we have following models- (i) Centralized network (ii) Distributed network (iii) Peer-to-peer network[3].

By centralized control we mean a node does not generate its own schedule; rather, it executes schedule generated by and downloaded from a central scheduler such as the base station. The node simply collects communication statics and forwards them to the central scheduler. On the other hand by distributed control we mean a node is autonomous. It schedules its own task and data processing. It also process request from its neighbor and the host. In a centralized network, the central controller generates the routing path and distributes them to each node; in distributed networks, each node builds its own routing information by talking with each other. Public network technology advances and increases the no of nodes with powerful PCs have helped shift the conventional client server model towards peer-to-peer (P2P) topologies.P2P leverages vast expanse of worldwide PC computing power, storage and connectivity. In P2P each node (peer) acts symmetrically as client and server. Large numbers of peers get together dynamically in an ad hoc manner and share information in large scale distributed environments without centralized coordination.

The P2P information retrieval (IR) environment we discuss here assumes that each peer has a database (or collection) of documents (text, audio, video or other semi structure documents) that is shares in the network [4]. A node searches full information by sending query messages that contain sets of keywords to its peers.

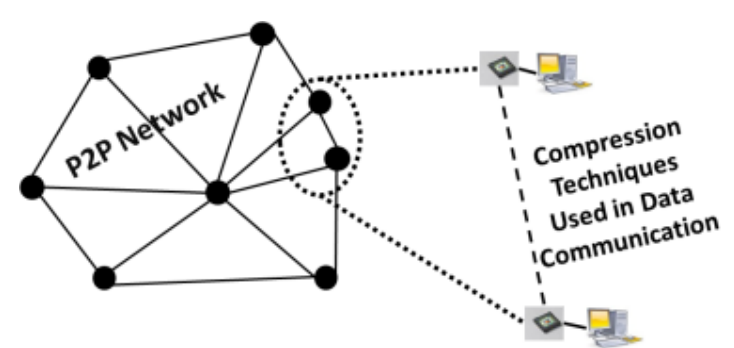

Figure 2: Data Compression in WSN P2P Network

A peer receiving a query message compares the similarity of the query against its document collection. Typically, this involves identifying documents that contain the set of query keywords. If the evaluation is successful, the peer sends a reply message that contains pointers to the matching document.

Figure 2 explains the use of various compression techniques for reducing the energy consumption in $\mathrm{p} 2 \mathrm{p}$ based wireless sensor network (WSN).

\section{Energy saving using Compression}

The general assumptions for sending the data over network are that nodes are given time slots in which they are known to not interface with one another. The nodes also know their own locations, and the locations of other nodes in the network previously.

For decreasing the power consumption there are various compression techniques. The sensor network without data compression will serve to be the baseline in performance and power consumption. The data is compressed before sending it out to reduce network transmission time and reduce the total application elapsed time. But it also increases the local processing time by introducing the compression overhead.

Whether or not an application can benefit from data compression depends on the tradeoff between the reduction of network transmission time and the increases of local processing.

According to data types various compression techniques are available as shown in figure 3. We are explaining some of them considering only text files. 


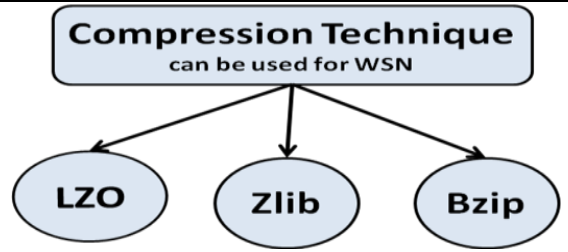

Figure 3: Types of Compression Techniques

LZO [6] supports overlapping compression and in-place decompression. It is a block compression algorithm - it compresses and decompresses a block of data.

Zlib's [7] compression method emits compressed data as a sequence of blocks. Various block types are allowed, one of which is stored blocks - these are simply composed of the raw input data plus a few header byte The parallel Bzip2[8] algorithm works by taking the blocks of input data and running them through the Burrows-Wheeler Transform(BWT) simultaneously on multiple processors using p threads.

Unfortunately, there are several limitations in the use of compression for efficient data communication First, compression techniques vary in their performance characteristics: compressed size, compression time, and decompression time.

Second, compression performance is reliant upon the performance and availability of the underlying resources, e.g., CPU loads, network bandwidth and latency.

\section{Choosing Best Compression Technique}

The best compression technique for the user will depend on the network it is connected to. Furthermore, as the load changes at communication end-points or on the network itself, the best- performing compression technique for the same network also changes. These limitations make it increasingly difficult for end users to identify the best compression technique in all circumstances. Adaptive Compression Environment (ACE) will identify the best compression technique automatically and transparently and applies compression onthe fly. ACE selects between a number of well-known, competitive, compression techniques including Bzip, Zlib, and LZO [5].

Now after compression the actual transmission of data takes place. By using compression we have reduced the data size which we have to transmit and receive so the energy consumption is also reduce as to transmit/receive sensor have to be in active mode for less time as compared to transmitting data without compression.

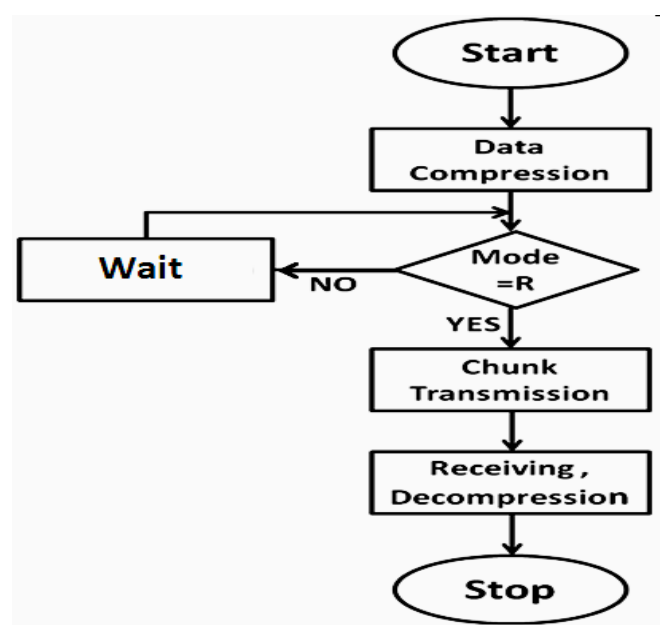

Algorithm:

Figure 4: Proposed energy saving solution in P2P oriented WSN

Step1: Start

Step2: Sender Compress the data by using best technique and divide it into chunks.

Step3: Check whether sensor is in R mode. if yes go to step 5

Step 4: Wait $(\mathrm{Et}+\mathrm{Ts}(\mathrm{n}))$ and go to Step 3

Step 5: Sensor transmits the chunks.

Step 6: Receiver receive and decompress the data and use it for further work.

Step7: Stop 


\section{Conclusion}

In the recent scenario of Wireless Sensor Networks, many new technologies are there for saving the energy of sensors. In this Paper, a solution of compression technology is proposed in peer to peer network. In the near future, much advancement will be made in this work and an appropriate solution will be attained.

\section{References}

[1] I.F.Akyildiz,W.Su,Y.Sankarasubramaniam and E. Cayirici, “A Survey on sensor Networks,” IEEE Comm. Mag., Aug 2002, pp 102-114.

[2] C.-F. Chiasserini and M. Garetto, "Wireless Sensor Networks,” IEEE INFOCOM 2004.

[3] A.Saroliya,"InvestigationAnalysistofindtheSecurityVulnerabilitiesinDHTOrientedPeertoPeerNetwork", Proceedingsofthe1 stInternational Conference on Parallel, istributed and Grid Computing (PDGC-2010), 978-1-4244-76732/10/\$26.00 @2010 IEEE.

[4] Demetrios Zeinalipour-Yazti,Vana Kalogeraki, And Dimitrios Gunopulos," Information Retrieval Techniques For Peer to Peer," 1521-9615/04/\$20.00 @ 2004IEEE.

[5] Chandra Krintz and Sezgin Sucu, "AdaptiveOn-the-FlyCompression," 1045-9219/06/\$20.00 @ 2006 IEEE

[6] Lempel-Ziv-Oberhumer(LZO) Compression,2005, www.oberhumer.com/opensource/lzop/.

[7] ZLib compression library, www.gzip.org/zlib/+, 2005.

[8] BZIP Compression, http://sources.redhat com/bzip2/, 2005. 